Inge Ziegler

\section{Worum geht es in der \\ Psychosomatischen Energetik?}

Die PSE unterscheidet vier Ebenen des feinstofflichen Feldes mit unterschiedlichen stofflichen und seelischen Qualitäten, deren Energie im Rahmen der PSE mithilfe eines speziell hierfür konzipierten Gerätes durch einen kinesiologischen Muskeltest getestet werden kann:

- Vitalkörper: Lebenskraft, Empfinden der eigenen Kraft und Belastbarkeit, Erdung;

- Emotionalkörper: Gefühle, Stimmungen, Abwehrsystem, Lernfähigkeit;

- Mentalkörper: Alltagstauglichkeit, Ich-Bewusstsein, Konzentration, Persönlichkeit;

- Kausalkörper: tiefes Unbewusstes, Feinfühligkeit, Intuition, Sensibilität, Kreativität, Kontakt «nach oben».

Von der Norm abweichende Werte deuten auf Störungen im energetischen System hin. Hinter einem Energiemangel auf einer der vier Ebenen findet man in der Regel einen oder mehrere Konflikte, die das zugehörige Energiezentrum (Chakra) blockieren und langfristig regelrecht als Energieräuber wirken. Insgesamt kennt die PSE 28 unterschiedliche

Aufgezeichnet von Dr. rer. nat. Inge Ziegler, Fachjournalistin im Auftrag der Internationalen Gesellschaft für Psychosomatische Energetik (IGPSE), nach einem Vortrag von Dr. med. Suzanne von Blumenthal (www.logosynthese.ch), ehemalige Chefärztin in der Graubündener Psychiatrie und Dr. med. FMH für Psychiatrie und Psychotherapie, anlässlich des 13. Expertentreffens der IGPSE vom 27.05.2017. Literatur bei der Verfasserin.

\title{
Psychosomatische Energetik in der Psychiatrie
}

«Eignet sich die Psychosomatische Energetik (PSE) als Standardverfahren für Diagnostik und Therapie in der Psychiatrie?», so lautete die Kernfrage, der sich Dr. Suzanne von Blumenthal in ihrem Vortrag widmete (Abb. 1). Nach 8 Jahren PSE in der Bündner Psychiatrieklinik, wo sie sich als Chefärztin die Aufgabe stellte, komplementärmedizinische Verfahren in die Psychiatrie einzuführen, kommt sie zu einem eindeutigen Ergebnis ...

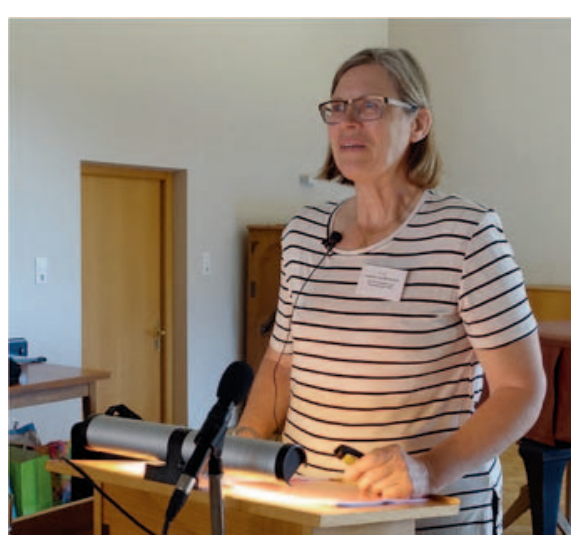

Abb. 1. Dr. med. Suzanne von Blumenthal bei ihrem Vortrag.

Konfliktthemen, die zunächst mit entsprechenden Testampullen eruiert und dann mittels spezieller homöopathischer Komplexmittel aufgelöst werden.

\section{Um welche Erkrankungen geht es?}

In der Psychiatrie hat Dr. von Blumenthal mit einer Vielzahl psychiatrischer Erkrankungen zu tun. Im Folgenden sollen einige wesentliche Erkrankungen mit ihren wichtigsten Symptomen und Erfahrungen aus der PSE kurz vorgestellt werden.

\section{Depression}

Symptome:

- Verminderte Belastbarkeit, sich schlapp fühlen, Schlafstörungen;

- Stimmungsschwankungen, verminderte Freude und Lust, auch Scham- und Schuldgefühle;

- Vergesslichkeit, sich verändert erleben, Grübelneigung;

- alles ist starr, «Austausch» reduziert, völlige Einsamkeit.

Typischer Befund bei der PSE:

- Vitalwert: in der Regel deutlich erniedrigt;

- Emotionalwert: häufig sehr niedrig (10-20\% statt einem Normalwert von $100 \%$ );

- Mentalwert: bei schweren Depressionen oft erniedrigt;

- Kausalwert: häufig erniedrigt; wenn alles erstarrt ist, kann er auch hoch sein.

Die niedrigen Vital- und Emotionalwerte decken sich häufig mit dem Gefühl der Leere dieser Patienten, die oft nichts mehr richtig spüren und sich als bedeutungslos empfinden. Nach guten Erfolgen der PSE bei Depressionen ist derzeit eine Doppelblindstudie in der Bündner Psychiatrie zur Frage geplant, wie gut sich Depressionen mit der PSE behandeln lassen.

\section{KARGER}

(๑) 2017 S. Karger GmbH, Freiburg

Fax +497614520714 


\section{Psychose}

Symptome:

- Körperliche Anspannung, Bewegungsdrang oder Erstarrung, wenig Erdung;

- Angst, Gereiztheit, Wut;

- Gedankendrängen, Gedankenflucht, Wahrnehmungsveränderungen, Ich-Störung;

- «Übersensibilität», veränderte Intuition, erhöhte Kreativität.

Typischer Befund bei der PSE:

- Vitalwert: oft erniedrigt;

- Mentalwert: oft erniedrigt;

- Kausalwert: oft hoch, 70\% oder sogar höher.

Bei Schizophrenie, einer chronischen Unterform der Psychose, spielen häufig energetische Blockaden in den Chakren 1 (Sexualchakra) und 7 (Scheitelchakra), aber auch Angst und Stress eine Rolle. Ausdrücklich warnte Dr. von Blumenthal vor CannabisKonsum oder übermässigem Alkoholgenuss in jungen Jahren. Demnach kann Cannabis bis zum Abschluss der Hirnentwicklung im 25. Lebensjahr besonders grosse Schäden im Gehirn anrichten und durchaus eine Schizophrenie auslösen. Erstaunt zeigte sich die Ärztin über die Erfolge der PSE bei Schizophrenie. Auch wenn Schizophrenie-Patienten eher kritisch seien, könnte sie nur zum Einsatz der PSE bei Schizophrenie ermutigen.

\section{Angststörung}

Symptome:

- Körperlich angespannt, nervös, unruhig, körperliche Reaktionen;

- Angst, Stimmungsschwankungen;

- Konzentrationsstörung, Kontrollbedürfnis, Gedankenkreisen;

- erhöhte Sensibilität und Feinfühligkeit;

Typischer Befund bei der PSE:

- Vitalwert: oft erniedrigt 40-70;

- Emotionalwert: oft erniedrigt 40-70;

- Mentalwert: leicht erniedrigt 70-80. Insgesamt sind Angststörungen der Behandlung mit der PSE besonders gut zugänglich. Bei Angst und Unruhezuständen sind gemäss Dr. von Blu- menthal die PSE-Mittel Anxiovita ${ }^{\circledR}$ und Neurovita ${ }^{\circledR}$ ebenso gut wirksam wie Anxiolytika - nicht nur, wenn sie in der PSE-Diagnostik positiv getestet wurden. Zudem sollte die PSE bei schweren Störungen immer mit allopathischen Medikamenten ergänzt werden.

\section{Suchtmittelabhängigkeit}

Symptome:

- Körperliche Missempfindungen;

- wenig Erdung;

- Stimmungsschwankungen (30\% der Abhängigen haben eine Depression), Gereiztheit, Wut, Schamund Schuldgefühle;

- Wunsch nach einem wohligen $\mathrm{Zu}$ stand: «sorgenfrei», Ausblenden der Realität;

- sehr sensibel (decken ihre Gefühle daher gern mit Drogen zu), Kontakt vermeidend.

Typischer Befund bei der PSE:

- Mentalwert: kann unterschiedlich sein;

- Kausalwert: kann hoch oder tief sein.

Süchtige Menschen haben oft auch depressive Verstimmungen, die sich mit PSE gut behandeln lassen. Auch kann der Suchtdruck nach 2-3 Konfliktbehandlungen rückläufig sein. Insbesondere vegetative Beschwerden, die bei Suchtmittelentzug auftreten, werden gelindert.

\section{Essstörung}

Symptome:

- Körperliche Missempfindungen, Anspannung, innere Unruhe;

- Angst, fixes Körperbild, «Selbstverletzungen»;

- vermindertes Selbstbewusstsein, Glaubensmuster (z.B. Perfekt-Antreiber);

- sehr feinfühlig, kreativ.

Typischer Befund bei der PSE:

- Mentalwert: häufig erniedrigt 40-70.

Glaubensmuster, die bei Essstörungen häufig eine Rolle spielen, lassen sich mit der PSE gut auflösen. Unterstützend können gegebenenfalls
Einführungsseminar für Ärzte in Bad Ragaz

Unter der Leitung von Dr. med. Heike Fischer, Allgemeinärztin aus Berlin, sowie Dr. med. Suzanne von Blumenthal wird ein PSE-Basisseminar in Bad Ragaz, Bahnhofstrasse 38, in den Institutsräumen des Instituts für Logosynthese angeboten. Nähere Informationen erhalten Sie unter www.igpse.ch sowie beim Institut für Logosynthese.

Glaubenssätze oder Logosynthese eingesetzt werden.

Ein besonders ernstes Problem unter den Essstörungen ist die Anorexie - immerhin sterben 15\% der Betroffenen daran. Entsprechend gilt es zunächst, die Magersüchtigen irgendwie über die Zeit mit dem niedrigen Gewicht zu bringen. Die Behandlung selbst erweist sich bei der Anorexie als sehr langwierig - 1-2 Jahre reichen hier leider nicht aus.

\section{Demenz}

Symptome:

- Müdigkeit, Koordinationsstörungen;

- ärgerlich, traurig, Schamgefühle;

- verminderte Planungs- und Steuerungsfähigkeit, verminderte Orientierung, Persönlichkeit verändert sich, Gedankenwiederholungen, Rückzug;

- oft noch feinfühlig, sensitiv.

Typischer Befund bei der PSE:

- Inwieweit die Werte verändert sind, hängt stark von der Phase der Erkrankung ab.

Bei beginnender Demenz sind die Erfolge mit der PSE nach den Erfahrungen von Dr. von Blumenthal recht gut. Vegetative Begleitsymptome, Schlafstörungen und Müdigkeit bessern sich fast immer unter der PSE.

\section{Persönlichkeitsstörung}

Symptome:

- In der Regel wenige körperliche Reaktionen;

- wenige emotionale Reaktionen bis hin zu sehr heftigen Reaktionen (Borderline-Persönlichkeitsstörung); 
- sehr starre Glaubensmuster, feste Überzeugungen, Skepsis, Misstrauen;

- wenig sensibel bis hochsensibel. Typischer Befund bei der PSE:

- Vitalwert: meist normal;

- Emotionalwert: oft niedriger.

Patienten mit einer Persönlichkeitsstörung stehen der PSE in der Anfangsphase oft sehr skeptisch gegenüber. Wenn sie sich dennoch darauf einlassen, sind es oft vor allem die Angehörigen, die zuerst die therapiebedingten Veränderungen wahrnehmen. Insbesondere die für Persönlichkeitsstörungen typischen starren Glaubensmuster sind der PSE gut zugänglich.

\section{Was leistet die Psychosomatische Energetik in der Psychiatrie?}

\section{PSE als «Psychotherapie in \\ Tropfenform»}

Das vegetative Nervensystem ist bei vielen psychiatrischen Erkrankungen der Dreh- und Angelpunkt der Beschwerden. Eine besonders wichtige Struktur in diesem Zusammenhang ist der Hippocampus als eine zentrale Schaltstation des limbischen Systems, in dem unsere Erfahrungen gespeichert werden. Wenn man nun weiss, dass die PSE das vegetative Nervensystem mit seiner Verbindung $\mathrm{zu}$ den Chakra-Ebenen stabilisiert, wird leicht verständlich, wieso die PSE bei vielen psychiatrischen Störungen wie z.B. Ängsten und Depressionen wirksam ist. Tatsächlich können mit der PSE regelmässig Patienten emotional stabilisiert und vegetative Begleitsymptome abgeschwächt werden (Abb. 2).

\section{PSE plus Psychopharmaka?}

So gut die Erfolge der PSE in vielen Bereichen der Psychiatrie auch sind, bei mittelschweren bis schweren Störungen sowie schwerer Beeinträchtigung der Lebensqualität ist die zusätzliche Gabe von Psychopharmaka einfach notwendig. Glücklicherweise befruchten sich die beiden Ansätze

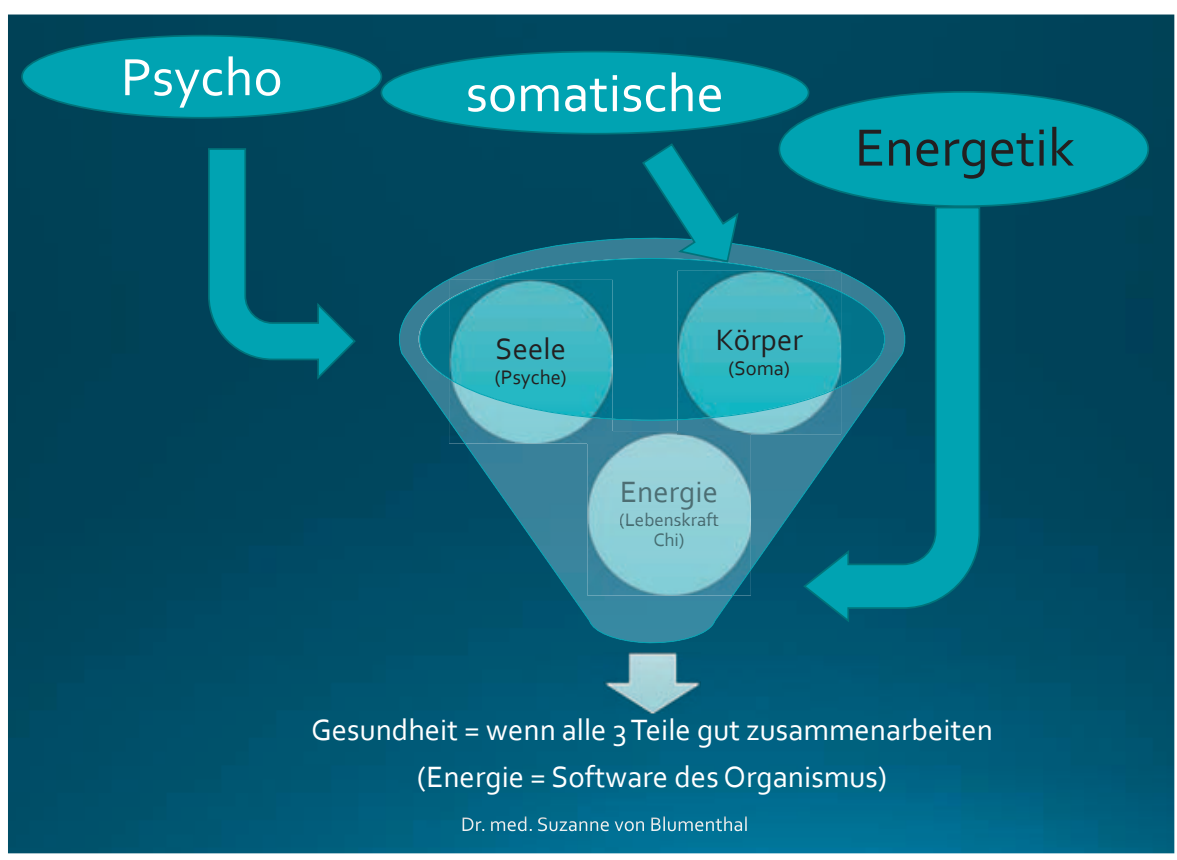

Abb. 2. Die Psychosomatische Energetik trägt dazu bei, dass Körper, Seele und Energie (Lebenskraft) ins Gleichgewicht kommen.

dabei gegenseitig. So kann man mit Psychopharmaka akute Phasen überbrücken, bis die PSE angeschlagen hat. Umgekehrt lässt sich die Wirkung der Pharmakotherapie deutlich steigern, indem man die PSE zur Medikamententestung einsetzt.

\section{Medikamententestung mit der PSE}

Unabhängig davon, ob es um Antibiotika oder Psychopharmaka geht: Laut Dr. von Blumenthal kommen schulmedizinische Medikamente im Allgemeinen auf eine Erfolgsquote von etwa $60 \%$. Gerade bei Medikamenten, die mit starken Nebenwirkungen behaftet sind, sowie bei schweren Erkrankungen, die einen hohen Leidensdruck bei den Patienten auslösen, kann diese Quote wohl kaum als zufriedenstellend betrachtet werden. Dank der Medikamententestung mit der PSE gelingt es Dr. von Blumenthal zu 95\%, ein wirksames Präparat auszuwählen! Gegenüber der «normalen» Erfolgsquote von $60 \%$ ist das immerhin eine Steigerung um weitere 35\%. Auf diese Weise können die Menge und Dauer der Medikation verringert, die Nebenwirkungen reduziert und die Akzeptanz der Therapie durch den Patienten erheblich verbessert werden. Grund genug für die Ärztin, heute keinen Patienten mehr ohne Medikamententestung einzustellen.

\section{Bessere Erfolge durch Kombination} mit der PSE

Gerade bei therapieresistenten oder schwierigen Patienten, bei denen die bisherige medikamentöse Therapie nicht zum gewünschten Erfolg geführt hat, kann die PSE der Schlüssel zum Erfolg werden. In der Klinik hat Dr. von Blumenthal ärztlichen Kollegen in solchen Fällen gerne Listen mit Psychopharmaka zur Verfügung gestellt, die in der PSE-Messung positiv testeten. Wenn diese sich auf den Versuch einliessen, waren sie oft erstaunt über die hohe Trefferquote. Dieses Vorgehen könnte auch für Ärzte und Heilpraktiker interessant sein, die selbst keine Psychopharmaka verordnen (dürfen).

Auch bei Patienten, die unter Umständen jeglicher medikamentösen Therapie skeptisch gegenüberstehen und ihre dringend notwendigen Medikamente nicht (regelmässig) nehmen, weil der Erfolg bislang ausgeblieben ist oder die Nebenwirkungen zu stark wa- 
ren, kann die Akzeptanz für die medikamentöse Therapie deutlich gesteigert werden, wenn die Patienten erst mal spüren, dass es ihnen damit einfach besser geht. Daher ist es durchaus sinnvoll, Patienten zu bitten, die entsprechenden Präparate zur Testung mitzubringen.

Darüber hinaus kann die PSE bei Patienten eingesetzt werden, die einer normalen Psychotherapie eher ablehnend gegenüberstehen oder nicht so viel Zeit in die Behandlungstermine investieren wollen. Während für eine Psychotherapie alle 1-2 Wochen eine Sitzung nötig ist, reicht bei der PSE meist ein Abstand von 2-4 Monaten.

Wenn geopathische Belastungen den Therapieerfolg blockieren, lässt sich das ebenfalls mit der PSE austesten. Insbesondere bei sehr niedrigen Vitalwerten sollte dies mit der Testampulle Geovita ${ }^{\circledR}$ überprüft und gegebenenfalls geopathische Belastungen beseitigt werden.

Ein weiterer Vorteil der PSE liegt zweifelsohne darin, dass man durch sie einen anderen Zugang zum Patienten gewinnt und so häufig eine Kommunikation auf einer anderen Ebene möglich wird. Das erleichtert natürlich die psychotherapeutische Arbeit. Hinzu kommt, dass durch die Klärung einzelner Themen im Rahmen der PSE-Therapie nach und nach andere Themen klarer werden und dann besser bearbeitet werden können. Genutzt werden kann dies z.B. in Fällen, in denen die Verhaltenstherapie gerade keine Fortschritte macht. Dann können mittels PSE Themen aktiviert werden, die dann wieder der Verhaltenstherapie zugänglich werden. In anderen
Tab. 1. Erfahrungen aus dem Praxisalltag mit der Psychosomatischen Energetik (PSE)

Fällen kann die PSE genutzt werden, um Patienten erst einmal so weit zu stabilisieren, dass sie einer Psychotherapie überhaupt erst zugänglich werden.

Umbauten im Gehirn brauchen Zeit Unser Gehirn ist sehr plastisch. Durch Lernen und Abspeicherung von Erfahrungen verändern wir es permanent. Entsprechend werden Nervenverbindungen im Gehirn (Dendriten) angelegt oder Botenstoffe an bestimmten Stellen ausgeschüttet. Für Patienten mit psychiatrischen Störungen heisst das zugleich, dass sie ihre psychische Gesundheit erst wieder erlernen müssen und ihr Gehirn entsprechend umgebaut werden muss. Entsprechend ist beim Einsatz der PSE in der Psychiatrie schon etwas Geduld gefragt. 2-5 Jahre rechnet die Ärztin in der Regel für eine PSE-Therapie. In Einzelfällen kann es auch deutlich schneller gehen, wie der Fall einer Patientin mit Zahnarztangst zeigt, der binnen nur einer Woche geholfen werden konnte. Bis es so weit ist, sollte der Patient bei Bedarf mit Psychopharmaka unterstützt werden (Tab. 1).

\section{Fazit}

Die Erfahrungen aus dem Klinikalltag haben Dr. von Blumenthal darin bestärkt, mit der PSE auf dem richtigen Weg zu sein. Mit der PSE lassen sich Energieblockaden lösen, die Patienten emotional stabilisieren, vegetative Beschwerden bessern und die persönliche Entwicklung unterstützen. Die eingangs gestellte Frage, ob sich die PSE als Standardverfahren für Diagnostik und Therapie eignet, kann nach Ansicht der Ärztin eindeutig mit Ja beantwortet werden. Bei ihrer eigenen psychotherapeutischen Arbeit hat die PSE die «normale» Diagnostik sozusagen überflüssig gemacht. Darüber hinaus hat sich die PSE sowohl als «Psychotherapie in Tröpfchenform» als auch zur Medikamententestung als überaus wertvoll erwiesen. Aufgrund dieser Möglichkeiten kommt Dr. von Blumenthal zu dem Schluss, dass die PSE immer noch viel zu wenig in der Psychiatrie genutzt wird. Sie jedenfalls möchte sie in ihrer täglichen Praxis nicht mehr missen. 